\title{
PRESENCIA DE MOSQUITERO COMÚN (PHYLLOS- COPUS COLLYBITA) Y MOSQUITERO IBÉRICO (PHYLLOSCOPUS IBERICUS) EN LA PROVINCIA DE SALAMANCA
}

\author{
Antonio CEBALLOS BARBANCHO \\ Departamento de Geografía -Universidad de Salamanca
}

Recibido: 29/04/2011

Devuelto: $17 / 07 / 2011$

Aceptado: 16/09/2011

RESUMEN: El principal objetivo del presente trabajo es determinar la presencia de mosquitero común (Phylloscopus collybita) y mosquitero ibérico (Phylloscopus ibericus) en la provincia de Salamanca durante el período reproductor. Para ello se realizaron un total de 78 transectos lineales en hábitats potencialmente favorables para ambas especies. Los resultados obtenidos demuestran la presencia de ambas especies en la provincia de Salamanca, pero de forma escasa, puntual y dispersa, siempre en zonas con un ombroclima sub-húmedo/húmedo o en bosques de ribera azonales con un microclima específico.

PALABRAS CLAVE: mosquitero común, mosquitero ibérico, distribución, Salamanca.

PRESENCE OF COMMON CHIFFCHAFF (PHYLLOSCOPUS COLLYBITA) AND IBERIAN CHIFFCHAFF (PHYLLOSCOPUS IBERICUS) IN THE PROVINCE OF SALAMANCA

ABSTRACT: The major objective of the present work is to determine the presence of Common Chiffchaff (Phylloscopus collybita) and Iberian Chiffchaff (Phylloscopus ibericus) during the breeding season in the province of Salamanca. For this, a total of 78 linear transects were carried out in potential and favourable habitats for both species. The main results show the spatial occurrence of the two species in the province of Salamanca. This is, however, scarce, sporadic and spatially dispersed, both in areas under sub-humid/humid ombroclimatic conditions and in azonal riparian forest with specific microclimatic conditions.

KEY WORDS: Common Chiffchaff, Iberian Chiffchaff, distribution, Salamanca.

\section{INTRODUCCIÓN}

La separación a nivel de especie del mosquitero común (Phylloscopus collybita) y el mosquitero ibérico (Phylloscopus ibericus), a partir del trabajo de Helbig et AL. (1996), plantea en la actualidad el reto de diferenciar ambos taxones en aquellos territorios en donde coexisten. Con anterioridad a 
dicho trabajo, Phylloscopus collybita era considerado como una especie polítípica con una amplia distribución por el continente euroasiático y norte de África (FIGURA 1A). De acuerdo con CRAMP (1992) y BAKER (1997), la subespecie nominal collybita se distribuiría por Europa Occidental continental e Islas Británicas, extendiéndose hacia el norte por Dinamarca y sur de Suecia; por el sur alcanzaría Los Pirineos, expandiéndose por Italia, Sicilia, antigua Yugoslavia y Grecia; representando finalmente Bulgaria, Rumanía, Hungría y Polonia su límite oriental. La subespecie brehmii ocuparía la Península Ibérica, el suroeste de Francia y el noroeste de África.

Figura 1. Taxonomía de Phylloscopus collybita $y$ Phylloscopus ibericus antes y después de la separación de ambos a nivel de especie.

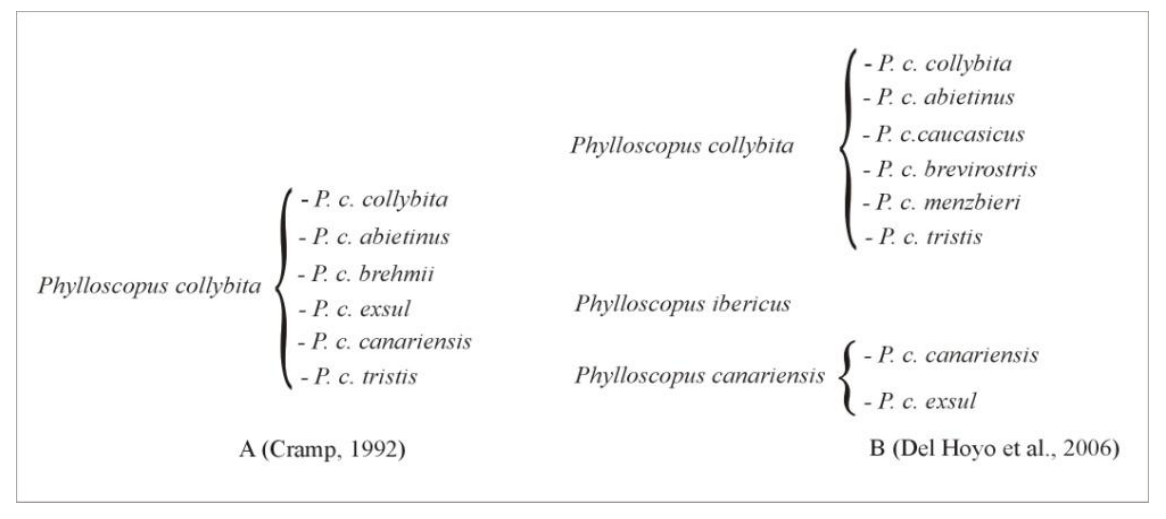

A partir del citado trabajo de HeLBIG et AL. (1996), y otros posteriores como los de SALOMON et AL. (1997) y HelBig et AL. (2001), Phylloscopus collybita brehmii es elevado a la categoría de especie, bajo la denominación de Phylloscopus ibericus, de acuerdo a criterios bioacústicos, morfológicos y de biología molecular, formando una superespecie junto a Phylloscopus collybita y Phylloscopus canariensis (DEL HOYO et AL. 2006 -FIGURA 1B-). El mosquitero común $(P$. collybita) seguirá siendo considerado una especie politípica, mientras que en el caso del mosquitero ibérico ( $P$. ibericus) SALOMON et $A L$. (2003) propusieron la diferenciación de dos subespecies ( $P$. ibericus ibericus y $P$. ibericus biscayensis), que de momento no ha sido ampliamente aceptada, que fue rebatida por ELÍAS (2004) y no ha sido recogida en el Handbook of the Birds of the World (DEL HOYO et AL., 2006).

Con anterioridad a la separación de ambos taxones a nivel de especie, trabajos como los de CRAMP (1992) y BAKER (1997) sugieren que durante la época reproductora sólo $P$. collybita brehmii se distribuiría por la Península Ibérica, mientras que $P$. collybita collybita se extendería por el continente europeo a partir de los Pirineos hacia el este. Durante la época invernal, $P$. $c$. 
brehmii migraría mayoritariamente a diversos países de la franja subsahariana africana, mientras que parte del contingente europeo de $P$. c . collybita invernaría en la franja meridional de Europa, acogiendo la Península Ibérica un número más que apreciable de aves. De ello se deduce que durante el invierno los mosquiteros presentes en la Península Ibérica serían collybita en un alto porcentaje, ya que brehmii migraría a África; durante los pasos migratorios ambas subespecies coincidirían; mientras que durante el período reproductor predominarían los individuos de la subespecie brehmii.

Una vez separadas ambas especies, se ha hecho un esfuerzo por precisar su distribución geográfica en relación con su fenología. Basándonos en las descripciones recopiladas en los trabajos de TELLERÍA et AL. (1999), CUESTA y BALMORI (2003), BALMORI y CUESTA (2003) y SANZ-ZUASTI y VELASCO (2005) hemos elaborado los mapas correspondientes a las FIGURAS 2A Y 2B para representar el grado de presencia de ambas especies en las provincias españolas peninsulares ${ }^{1}$ durante el período reproductor.

En el caso del mosquitero ibérico (P. ibericus), este se muestra localmente abundante en las provincias atlánticas y cántabras, incluidas en la región bioclimática eurosiberiana con precipitaciones abundantes, y bien repartidas durante el año, y temperaturas suaves (TELLERÍA et AL. 1999; RIVASMARTÍNEZ, 1987). La especie está ausente en las provincias orientales de la Península Ibérica. Curiosamente, entre los hábitats predilectos de la especie, DEL HOYO et AL. (2006) citan los coscojares (Quercus coccifera), siendo la coscoja un arbusto propio de terrenos más bien xéricos y calizos, que corresponden en el caso de la Península Ibérica a aquellas zonas en donde precisamente no se cita la especie como reproductora (arco levantino). Recientemente, CARRASCAL y PALOMINO (2008) han estimado una población de 1.060.535 individuos en el territorio peninsular español y han localizado las mayores densidades en los bosques más septentrionales, concretamente en riberas arboladas, robledales, pinares y encinares.

\footnotetext{
${ }^{1}$ La elaboración de los dos mapas correspondientes a la figura 2 se basa en una interpretación subjetiva de las descripciones de la distribución de ambas especies que aparecen en las obras señaladas. La clase localmente abundante responde a expresiones como: "en el norte de ... abunda mayoritariamente la especie ..." "en las provincias ... predomina ampliamente ...". La clase escaso y puntual responde a expresiones como "En ... predomina la especie X, aunque la especie Y también está presente de forma mucho más dispersa". La clase muy escaso y puntual responde a expresiones como: "presencia ocasional de ..." "presencia muy dispersa y escasa de ...". La clase ausente ha sido aplicada en aquellos casos en donde explícitamente se afirma que no hay registros en esa provincia. La clase sin datos ha sido aplicada cuando no se ha encontrado información relativa a esas provincias.
} 
La FIGURA 2 muestra que el mosquitero común (Phylloscopus collybita) es abundante en el cuadrante nororiental de la Península Ibérica, en donde ocupa montes arbolados y matorrales húmedos en las áreas montanas y es frecuente en bosques de riberas con Alnus y Salix en las tierras más bajas. Aparece repartido de forma muy discontinua, dispersa y escasa en el resto de provincias peninsulares españolas acantonándose en sotos fluviales altos y densos (De JuAna y VAREla, 2000; BALMORI et AL., 2002; CUESTA y BALMORI, 2003; Del HOYO et $A L ., 2006$ ). CARRASCAL y PALOMINO (2008) estiman una población de 1.665.122 individuos, con las mayores densidades en los bosques de la mitad norte peninsular, particularmente robledales, pinares y bosques de ribera.

Figura 2. Distribución de Phylloscopus ibericus (mapa izquierda) y Phylloscopus collybita (mapa derecha) en las provincias españolas peninsulares e Islas Baleares durante el período reproductor. Verde: localmente abundante; naranja: escaso y puntual; amarillo: muy escaso y puntual; gris: ausente; blanco: sin datos.

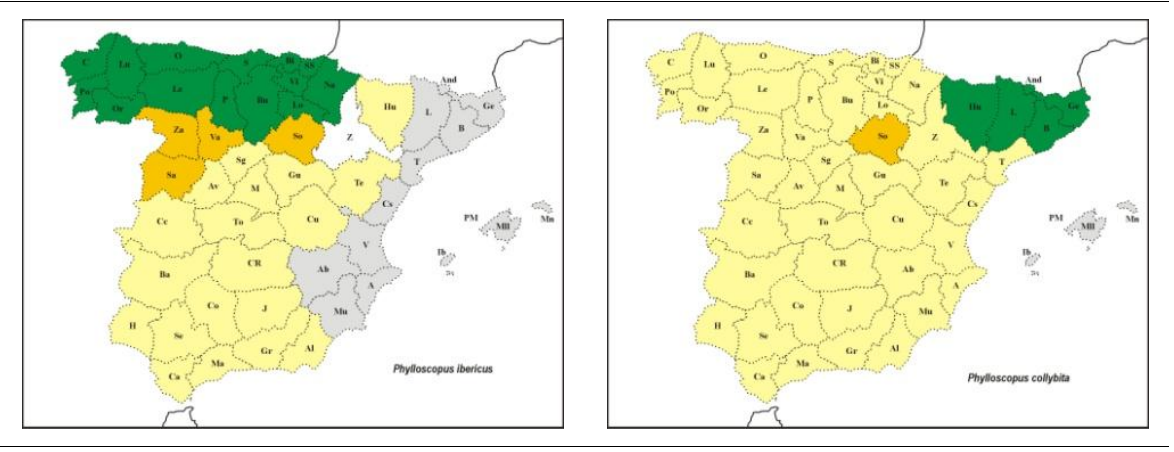

Aunque de los mapas correspondientes a la FIGURA 2 pudiera deducirse que en la Comunidad Autónoma de Castilla y León la presencia y densidad de $P$. ibericus es superior a la de $P$. collybita, conviene aclarar que según la estimación recientemente publicada de CARRASCAL y PALOMINO (2008) la población de $P$. collybita en Castilla y León, con 414.127 ejemplares, es más numerosa que la de $P$. ibericus con 307.775 aves.

En relación con el período invernal, $P$. ibericus migraría mayoritariamente a África (ONRUBIA et AL. 2003; CATRY et AL., 2005), mientras que una parte importante de la población europea de $P$. collybita pasaría el invierno en la Península Ibérica (DEL HOYO et AL., 2006).

Considerando la información comentada en los párrafos previos resulta evidente que existe un conocimiento limitado sobre la distribución de $P$. ibericus y $P$. collybita en gran parte del territorio peninsular. En el caso de Sa- 
lamanca, como se deduce de los anuarios ornitológicos de la provincia publicados hasta la fecha (BLANCO, 2004 y 2007), los trabajos publicados hasta ahora no ayudan a esclarecer la definición del estatus y fenología de ambas especies durante el período reproductor, ya que mientras para PERIS y CARnero (1988) y CUESTA et AL. (2003) Phylloscopus collybita (incluyendo ambas subespecies) ocuparía la mitad de la provincia, con serias evidencias de reproducción probable-segura, del trabajo de BALMORI et AL. (2002) solamente puede deducirse la presencia muy local y escasa de $P$. collybita.

Debido a ello, el objetivo principal del presente trabajo fue detectar la presencia de P. collybita y P. ibericus en la provincia de Salamanca como especies reproductoras, realizando un total de 78 transectos en los meses de mayo y junio de 2009 y 2010, en hábitats en principio favorables para ambas especies en el contexto de la región bioclimática mediterránea peninsular. El análisis y discusión de los resultados permitieron elaborar una sencilla hipótesis de trabajo con el objetivo de clarificar el estatus y fenología de ambas especies en el territorio provincial.

\section{II. ÁREA DE ESTUDIO}

La provincia de Salamanca ocupa una superficie de $12.350 \mathrm{~km}^{2}$ en la zona centro-occidental de la Península Ibérica. Desde un punto de vista fisiográfico pueden diferenciarse tres grandes unidades: i) las montañas y sierras del sur de la provincia que forman parte del Sistema Central; ii) las penillanuras de los sectores oeste y central y iii) los terrenos sedimentarios del cuadrante nororiental. La temperatura media de la provincia oscila en torno a los $12^{\circ} \mathrm{C}$, incluyéndose la mayor parte de la provincia por debajo de la isoterma de los $13^{\circ} \mathrm{C}$ o valor umbral que separa los pisos bioclimáticos meso- y supramediterráneo. Por encima de este valor tendríamos el paraje de Los Arribes del Duero, que ya pertenecería al ámbito bioclimático del piso mesomediterráneo. La precipitación media de la provincia oscila en torno a los $634 \mathrm{~mm}$, con un marcado contraste entre las precipitaciones registradas en las sierras del sur, en algunos enclaves superiores a los $1.500 \mathrm{~mm}$ anuales, y los menos de $375 \mathrm{~mm}$ que se miden anualmente por término medio en el cuadrante nororiental. Ello se traduce en una variedad de ombroclimas (húmedo, subhúmedo y seco) distribuidos a lo largo del territorio.

La vegetación potencial de la provincia, grosso modo, estaría representada por i) formaciones esclerófilas (principalmente de Quercus ilex ballota) en el cuadrante nororiental y sector central occidental del territorio provincial; ii) formaciones marcescentes de melojos (Quercus pyrenaica) y quejigos (Quercus faginea) en el cuadrante noroccidental y piedemontes serranos; iii) bosque mixto de caducifolios, marcescentes y coníferas en las montañas y 
sierras del sur; iv) matorral supraforestal en las zonas montanas más altas, y v) vegetación mediterránea termófila, tanto en Los Arribes como en el Valle del Alagón.

La red hidrográfica está articulada, salvo en el caso de la cabecera del río Alagón, por una serie de afluentes directos del Duero, que diseccionan la provincia en dirección SE-NO: Tormes, Huebra, Yeltes y Águeda. Estos ríos en muchos casos están flanqueados por formaciones boscosas, con diferente grado de complejidad, con Populus sp, Fraxinus angustifolia, Salix sp, Alnus glutinosa, etc. en el estrato arbóreo y Rubus sp, Rosa sp, Sambucus sp, Crataegus sp, Urtica sp, etc. en el nivel del sotobosque.

\section{METODOLOGÍA}

Para la toma de datos se realizaron un total de 78 transectos, en hábitats potencialmente favorables para $P$. collybita y $P$. ibericus, distribuidos en distintas unidades paisajísticas y bioclimáticas de la provincia de Salamanca (PERIS y CARNERO, 1988; RIVAS-MARTÍNEZ, 1987). Cada transecto consistió en un recorrido lineal de 300 metros tratando de identificar visual y/o acústicamente la presencia de mosquiteros. En todos los casos se reprodujo el canto de ambas especies para estimular la respuesta de las aves. Cada transecto fue georreferenciado con GPS (modelo $60 \mathrm{C}$ de Garmin), seleccionando el datum WSG 1984 y expresando las coordenadas en grados y minutos decimales.

Debido a que el objetivo del trabajo era diagnosticar la presencia de ambas especies en la provincia de Salamanca como reproductoras, el período de muestreo se restringió a las ocho semanas comprendidas entre el 1 de mayo y el 30 de junio con el propósito de evitar a individuos en paso migratorio. El trabajo de campo se distribuyó en dos campañas realizadas en 2009 y 2010. En el año 2009 se prospectaron principalmente los bosques de ribera, guiados por la conclusiones del trabajo de BALMORI et $A L$. (2002), con la realización de un total de 40 transectos. Teniendo en cuenta los resultados de esa primera campaña, en 2010 por un lado se siguieron prospectando otros bosques de ribera, pero también se muestrearon algunas zonas, en principio no favorables para las especies, pero de las que hay referencias documentales que acreditan su distribución en las mismas (PERIS y CARNERO, 1988; Programa SACRE SEO/BirdLife).

\subsection{Identificación de las dos especies}

Como consecuencia de la dificultad que entraña diferenciar visualmente ambas especies en el campo atendiendo a sus caracteres morfológicos, se utilizó el canto como principal criterio de identificación, grabando distintas pis- 
tas como documento testimonial. De acuerdo con CRAMP (1992), el canto de Phylloscopus collybita habitualmente consiste en una estrofa de duración variable, rítmica con una alternancia irregular de dos notas claras y enfáticas, una de ellas marcadamente más pronunciada: "chiff-chaff-chaff-chiff-chaffchiff-chiff", a menudo precedida o seguida de algunas notas bajas y vibrantes. El canto correspondiente a Phylloscopus ibericus consiste esencialmente en una frase compuesta por tres secciones ("tit-tit-tit-tit-tit-tswee-tswee-chitit-it-it-it"), acelerando ostensiblemente el ritmo en la última parte. No obstante, si el observador tiene cierta experiencia en ambas especies, y tiene la posibilidad de ver detenidamente y fotografiar las aves, sí es posible identificarlas estudiando los rasgos morfológicos que a continuación se detallan (ONRUBIA et AL., 2003; MCELWEE, 2010; SVENSON et AL. 2010).

Figura 3. Izquierda: mosquitero ibérico (Phylloscopus ibericus). Derecha: mosquitero común (Phylloscopus collybita). Los números indican los rasgos morfológicos que hay que considerar para proceder a una identificación positiva de ambas especie (ver texto principal). Fotos: Antonio Ceballos Barbancho (P. Ibericus); Juan Sagardía Pradera (P. collybita).

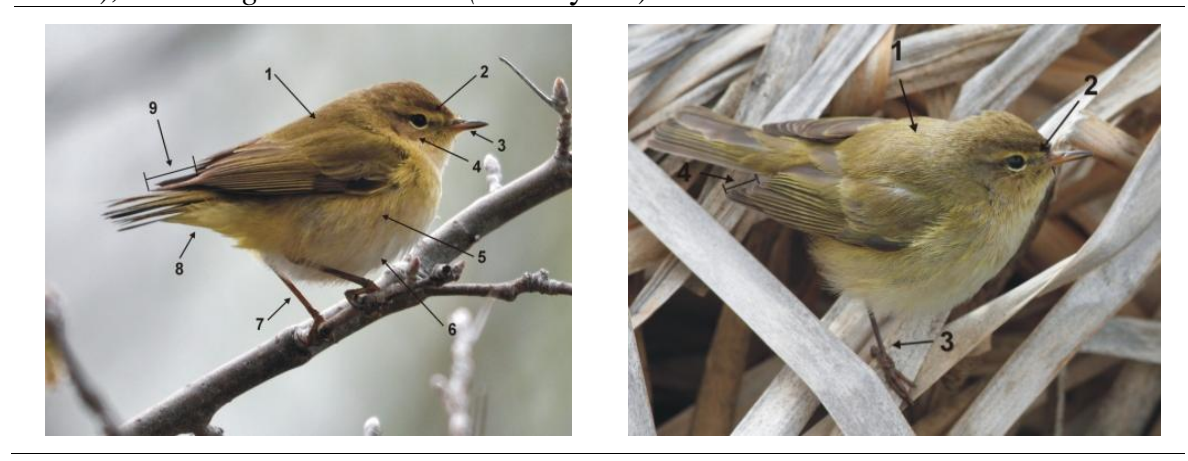

En el caso de P. ibericus habría que considerar una serie de caracteres de diagnóstico que aparecen señalados en la fotografía (FIGURA 3 izquierda) y que se enumeran a continuación: 1) tonalidad del manto más verdosa que pardo-grisácea; 2) parte frontal de la lista superciliar amarilla; 3 ) pico de color pálido, con tonalidad marrón-anaranjada en la mandíbula inferior; 4) mejilla poco marcada; 5) flancos amarillentos que contrastan con el blanco puro de la parte ventral (6), 7) patas de color marrón oscuro (no negras) algo más claro en los pies; 8) color amarillento en las infracoberteras caudales; 9) proyección de las primarias con una longitud intermedia entre Phylloscopus collybita y $P$. trochillus. En el caso de $P$. collybita, en comparación con $P$. ibericus, la tonalidad general del plumaje es más apagada. De manera más concreta, en la FIGURA 3 derecha pueden apreciarse los siguientes rasgos: 1) tonalidad del manto verde-grisácea; 2) lista superciliar bastante corta e indis- 
tinta, con ausencia de amarillo en la parte frontal de la misma; 3) patas de color muy oscuro (negruzcas), 4) proyección primaria corta.

\subsection{Descripción del hábitat}

En cada recorrido se realizó una descripción de los distintos hábitats, especialmente los bosques de ribera, atendiendo a criterios como la altura del arbolado, anchura del bosque, continuidad lineal del bosque y existencia o no de sotobosque (estrato arbustivo y herbáceo alto).

Para tratar de expresar cuantitativamente el grado de complejidad estructural de un determinado bosque aplicamos la siguiente tabla de clases:

\begin{tabular}{clllcc}
\hline Continuidad Lineal & \multicolumn{3}{c}{ Perfil transversal } & Altura del arbolado \\
\hline 1 & $\begin{array}{l}\text { Alta (hilera de árboles } \\
\text { sin huecos) }\end{array}$ & $\begin{array}{l}\text { Alto (anchura del } \\
\text { bosque }>10 \mathrm{~m})\end{array}$ & Alto $(>20 \mathrm{~m})$ \\
\hline 2 & $\begin{array}{l}\text { Media (pequeños cla- } \\
\text { ros a lo largo de la hi- } \\
\text { lera) }\end{array}$ & $\begin{array}{l}\text { Medio (anchura del } \\
\text { bosque entre 5 y 10 } \\
\text { m) }\end{array}$ & Medio $(15-20 \mathrm{~m})$ & \\
\hline $\begin{array}{l}\text { Baja (claros superio- } \\
\text { res a 5 m en línea) }\end{array}$ & 3 & $\begin{array}{l}\text { Bajo (anchura del } \\
\text { bosque }<5 \mathrm{~m})\end{array}$ & Bajo (<15 m) \\
\hline
\end{tabular}

Sumando los valores correspondientes a cada característica estructural podemos definir cuantitativamente cada tipo de bosque dentro de una escala que iría de 3 (bosque muy complejo: hilera de árboles sin huecos, anchura superior a $10 \mathrm{~m}$ y altura del arbolado superior a $20 \mathrm{~m}$ ) a 9 (bosque muy poco complejo: linealmente discontinuo con claros superiores a $5 \mathrm{~m}$, anchura inferior a $5 \mathrm{~m}$ y arbolado inferior a $15 \mathrm{~m}$ ). Nuestra hipótesis es que los bosques complejos tendrán capacidad de modificar las características ambientales del medio, principalmente las climáticas, ofreciendo un hábitat adecuado para las dos especies de mosquiteros estudiadas, mientras que los bosques muy poco complejos en principio serán menos aptos al no poder crear islas microclimáticas para las especies objeto de estudio (STERLING, 1996).

\section{RESULTADOS Y DISCUSIÓN}

En el mapa correspondiente a la figura 4 aparece representada la localización geográfica de los 78 transectos realizados: 66 corresponden a bosques de ribera con una composición florística y complejidad estructural diversa, 6 a melojares con sotobosque, 3 a encinares-quejigares y 3 a manchas de fresnos y chopos no asociadas a cursos de agua. La distribución de los transectos se hizo de acuerdo a los hábitats potenciales para ambas especies en la provincia, según las fuentes bibliográficas consultadas tal y como se expuso en la introducción. Ello explica la ausencia de puntos en el área central de la 
provincia, superficie ocupada por campos de cultivo y encinares con una densidad baja de arbolado, generalmente afectada por un régimen ombroclimático seco $(<600 \mathrm{~mm})$.

En 73 transectos no se obtuvo ningún tipo de contacto con las dos especies prospectadas, en 3 casos se registró la presencia únicamente de $P$. collybita, en 1 caso se documentó la presencia de las dos especies ( $P$. ibericus y P. collybita) en el mismo transecto y en el transecto restante no pudo identificarse de forma inequívoca la especie de mosquitero vista en el campo, ya que no cantó, aunque los rasgos morfológicos apuntaban a $P$. collybita.

Una información detallada sobre la localización y características mesológicas de los 5 transectos con resultado positivo es recogida en las TABLAS 1 y 2. Aunque una población de 5 casos es insuficiente para cualquier tipo de inferencia estadística, es apreciable que en aquellas zonas con un ombroclima seco todos los contactos se produjeron en bosques de ribera con un nivel de complejidad alto y en ríos con caudal permanente, debido a la capacidad de ese tipo de hábitat de garantizar unas condiciones microclimáticas aptas para ambas especies de mosquiteros, mientras que en los dos transectos con ombroclima húmedo parece que la dependencia de bosques riparios complejos es menor. En este sentido podría sugerirse que en el espacio geográfico de la provincia de Salamanca $P$. collybita y $P$. ibericus concurren espacialmente de forma muy discontinua en aquellas zonas con precipitaciones totales anuales comparables a las del tercio norte peninsular y en el caso de que estas decrezcan buscan enclaves microclimáticos como los bosques de ribera de estructura compleja. Este razonamiento se corresponde con las pautas de interpretación del hábitat para ambas especies en el territorio español peninsular en donde ambos mosquiteros ocuparían principalmente bosques caducifolios, especialmente de robles, hayas, abedules, alisos, etc. Conforme el clima se "mediterraneiza" su presencia es mucho más escasa, acantonándose en aquellas zonas que garanticen unas condiciones bioclimáticas comparables a las eurosiberianas: áreas montanas y umbrías supramediterráneas; bosques termomediterráneos de alcornoques y quejigos con ombroclima húmedo en las provincias del suroeste; o bien en bosques de riberas con carácter azonal (DE JUANA y VARELA, 2000; BALMORI et AL., 2002; CUESTA y BALMORI, 2003; SALOMON et AL. 2003; DEL HOYO et AL. 2006).

Las FIGURAS 5 y 6 muestran la distribución de los transectos con resultado negativo atendiendo a las clases de complejidad estructural del bosque y de ombroclimas respectivamente. De acuerdo con la información que recogen, en un $58,9 \%$ de los transectos con resultado negativo, pero con condiciones ambientales aptas para ambas especies, bien por situarse en una zona con ombroclima húmedo (> $600 \mathrm{~mm}$ ) o bien por tratarse de un bosque con es- 
Figura 4. Localización de los transectos realizados $(n=78)$. Puntos negros y grises: resultado negativo en 2009 y 2010 respectivamente $(n=73)$; puntos verdes: identificación positiva de de P. collybita $(n=3)$; punto rojo: identificación positiva de ambas especies $(n=1)$; punto azul marino: presencia de Phylloscopus sin identificar $(n=1)$. Otras fuentes: cuadrados azules: citas de P. collybita incluidas en la base de datos del programa Sacre tomadas en diferentes años entre el 1 de mayo y el 30 de junio. Triángulo azul: cita de P. collybita publicada por BALMORI et AL. (2002).

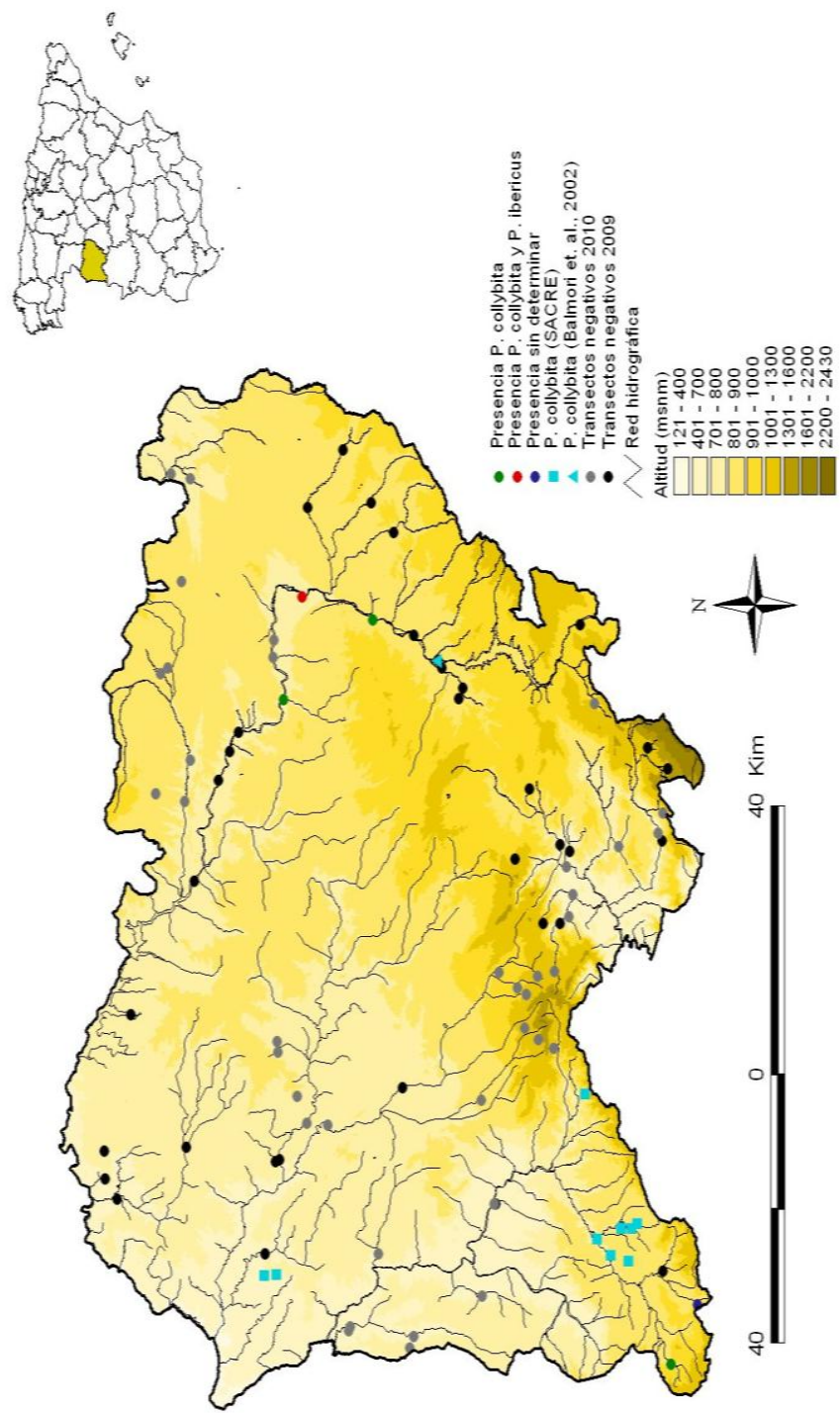


tructura compleja (clases 3 y 4), no detectamos su presencia. Considerando la totalidad de transectos con condiciones aptas $(n=48)$, detectamos la presencia de ambas especies en un 10,4\% de los casos, lo cual con mucha prudencia sugiere la posibilidad de considerar ambas especies como reproductoras muy escasas y con una distribución muy irregular y dispersa por la provincia.

Tabla 1. Localización geográfica de los transectos con resultado positivo.

\begin{tabular}{|c|c|c|c|c|c|c|c|c|}
\hline$I D$ & Fecha & Localidad & Rio $/ A^{\circ}$ & $\begin{array}{l}\text { Long. (grados, } \\
\text { min. dec.) }\end{array}$ & $\begin{array}{l}\text { Lat. (grados, } \\
\text { min. dec.) }\end{array}$ & $\begin{array}{c}\text { Ph. } \\
\text { ibericus }\end{array}$ & $\begin{array}{c}\text { Ph. } \\
\text { collybita }\end{array}$ & $\underset{\text { Sin }}{\text { identificar }}$ \\
\hline 11 & $08 / 05 / 09$ & Salamanca & Tormes & $5^{\circ} 40.187^{\prime} \mathrm{O}$ & $40^{\circ} 57.495^{\prime} \mathrm{N}$ & & + & \\
\hline 12 & $15 / 05 / 09$ & Alba de Tormes & Tormes & $5^{\circ} 31.373^{\prime} \mathrm{O}$ & $40^{\circ} 49.087^{\prime} \mathrm{N}$ & & + & \\
\hline 21 & $18 / 05 / 09$ & Encinas de Abajo & Tormes & $5^{\circ} 29.166^{\prime} \mathrm{O}$ & $40^{\circ} 55.925^{\prime} \mathrm{N}$ & + & + & \\
\hline 27 & $22 / 06 / 09$ & Navasfrías & Águeda & $6^{\circ} 48.475^{\prime} \mathrm{O}$ & $40^{\circ} 18.323^{\prime} \mathrm{N}$ & & + & \\
\hline 28 & $22 / 06 / 09$ & El Payo & Perosin & $6^{\circ} 42.074 ' \mathrm{O}$ & $40^{\circ} 15.974^{\prime} \mathrm{N}$ & & & + \\
\hline
\end{tabular}

Tabla 2. Características mesológicas de los transectos con resultado positivo.

\begin{tabular}{|c|c|c|c|c|c|c|c|c|}
\hline$I D$ & Fecha & Especies vegetales & $\begin{array}{c}\text { Complejidad } \\
\text { Estructural }\end{array}$ & $\begin{array}{c}\text { Piso } \\
\text { bioclimático }\end{array}$ & Ombroclima & $\begin{array}{c}\text { Ph. } \\
\text { ibericus }\end{array}$ & $\begin{array}{c}\text { Ph. } \\
\text { collybita }\end{array}$ & $\begin{array}{c}\text { Sin } \\
\text { identificar }\end{array}$ \\
\hline 11 & $08 / 05 / 09$ & Salix sp., Populus sp. & 3 & Supramediterráneo & Seco & & + & \\
\hline 12 & $15 / 05 / 09$ & $\begin{array}{l}\text { Salix sp., Populus } \\
\text { sp., Alnus glutinosa }\end{array}$ & 4 & Supramediterráneo & Seco & & + & \\
\hline 21 & $18 / 05 / 09$ & Salix sp., Populus sp. & 4 & Supramediterráneo & Seco & + & + & \\
\hline 27 & $22 / 06 / 09$ & $\begin{array}{l}\text { Alnus glutinosa, } \\
\text { Fraxinus } \\
\text { angustifolia, Salix } \\
\text { sp., Populus sp. }\end{array}$ & 8 & Supramediterráneo & Húmedo & & + & \\
\hline 28 & $22 / 06 / 09$ & $\begin{array}{l}\text { Alnus glutinosa, Salix } \\
\text { sp., Quercus } \\
\text { pyrenaica }\end{array}$ & 7 & Supramediterráneo & Húmedo & & & + \\
\hline
\end{tabular}

Figura 5. Grado de complejidad estructural de los tramos de bosques de ribera prospectados con resultado negativo

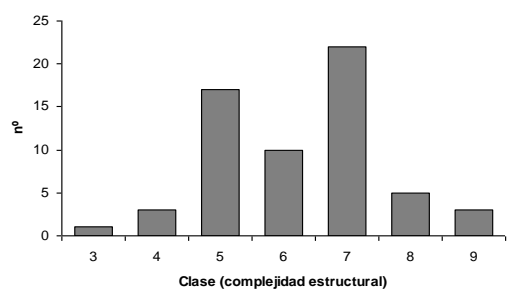

Figura 6. Ombroclimas de los tramos de bosques de ribera prospectados con resultado negativo.

Para definir el estatus de la(s) especie(s) en la provincia de Salamanca las únicas fuentes documentales existentes son, por orden cronológico, i) el Atlas ornitológico de la provincia de Salamanca (PERIS y CARNERO, 1988); 
ii) el libro de Raúl BuENo (2000) sobre las Aves de la Sierra de Béjar, iii) el artículo publicado en la revista Ardeola por BALMORI et AL. (2002) sobre la distribución de ambas especies en los bosques de ribera de Castilla y León; iv) las referencias que de la provincia, así como el mapa de presencia de la(s) especie(s), aparecen en el Atlas de Aves reproductoras de España (CUESTA y BALMORI, 2003), v) la síntesis que sobre el estatus de ambas especies recoge la nueva edición revisada y ampliada de la Guía de las aves de Castilla y León (SANZ-ZUASTI y VelASCO, 2005), vi) los dos volúmenes publicados hasta ahora del Anuario ornitológico de la provincia de Salamanca (BLANCO, 2004 y 2007) y, finalmente, vii) los datos incluidos en la base digital del programa SACRE coordinado por SEO/BirdLife.

Teniendo en cuenta los resultados obtenidos en 2009 y 2010, y los datos y/o conclusiones publicados en las citadas fuentes, podemos plantear la siguiente discusión a partir del desconocimiento sobre el estatus y fenología de ambas especies en la provincia de Salamanca (BLANCO, 2004 y 2007).

Antes de la separación de ambos taxones a nivel de especie, PERIS y CARNERO (1988) definieron $P$. collybita como posiblemente sedentario, con una distribución discontinua e irregular por gran parte del territorio de la provincia de Salamanca y ocupando terrenos arbolados con setos y maleza. La especie estaría presente en el 42,6 \% de las cuadrículas que cubrían la totalidad de la provincia durante el período reproductor, con una distribución relativamente regular por una diversidad de lo que ellos denominaron "regiones ornitogeográficas": complejo Arribes-robledal, estepa cerealista, encinar castellano y alta montaña. El mapa correspondiente al Atlas de las aves reproductoras de España (CUESTA y BALMORI, 2003) tampoco diferencia entre ambas especies, corroborando las conclusiones de PERIS y CARNERO (1988), ya que confirma la reproducción probable-segura de la especie en la mitad del territorio provincial para el período 1998-2002.

La Guía de aves de Castilla y León (SANZ-ZuASTI y VELASCO, 2005) sí separa ambas especies especificando, en el caso de Salamanca, que P. collybita estaría repartido por toda la provincia de forma irregular y dispersa, sobre todo en las dehesas centrales y occidentales, siendo abundante en invierno y mucho más puntual, escaso y localizado durante el período reproductor. En el caso de P. ibericus se limita a decir que es un reproductor escaso en la provincia. El estudio de BUENO (2000) tiene el interés, a pesar de su carácter local, de que prospecta una de las zonas que más se adecuan al hábitat potencial para ambas especies, la Sierra de Béjar. Se trata de un territorio de montaña, boscoso, y que goza de un ombroclima subhúmedo-húmedo. Según este autor, $P$. collybita sería una especie invernante, con la llegada de numerosos individuos a partir de finales de agosto, mientras $P$. ibericus sería 
una especie estival bien distribuida en esa zona. En el trabajo de BALMORI $e t$ $A L$. (2002), cuyo principal objetivo fue discernir la presencia y abundancia de ambas especies en los bosques de ribera de Castilla y León, en el caso de la provincia de Salamanca sólo detectaron la presencia de $P$. collybita en 1 de los 21 transectos que realizaron en la provincia, precisamente en un bosque de ribera en el río Tormes, con estructura compleja y en una zona de régimen pluviométrico seco.

Finalmente, aunque se trata de datos no publicados, actualmente en la provincia de Salamanca hay activas un total de 21 cuadrículas de 10 x $10 \mathrm{~km}$., gestionadas por voluntarios de SEO/BirdLife dentro del programa SACRE, que cubren aproximadamente el $17 \%$ del territorio provincial. En algunos casos tienen registros desde 2001, por lo que habría una serie interanual de 10 años. Analizando dicha base de datos se ha comprobado que en 7 cuadrículas (33\%) hay registros de $P$. collybita entre el 1 de mayo y el 30 de junio, no obstante habría que mencionar que las citas no tienen una frecuencia anual y existen años en los que no se cita la especie. En el caso de $P$. ibericus no se ha encontrado ninguna cita, bien porque los observadores consideren que se trata de una subespecie de $P$. collybita (aunque la propia base de datos sí distinga ambas especies) o bien porque realmente todas las citas correspondan a $P$. collybita.

Siguiendo principalmente el trabajo de BALMORI et AL. (2002) la primera campaña de campo, llevada a cabo en 2009 con un total de 40 transectos, se centró exclusivamente en la prospección de bosques de ribera en el territorio provincial (puntos negros correspondientes al mapa de la FIGURA 4). Ello permitió i) confirmar las conclusiones del citado trabajo, ii) constatar la presencia de los dos taxones en la provincia y iii) definir provisionalmente esta como puntual, dispersa y escasa. Considerando estos primeros resultados, y los trabajos de PERIS y CARNERo (1988), BUENO (2000) y CUESTA y BALMORI (2003), en 2010 una parte de los transectos realizados fue dirigida a prospectar las "regiones ornitogeográficas" en donde los citados autores incidían en que las especies eran más frecuente con un resultado negativo. Es más, incluyendo los bosques de ribera, en ninguno de los transectos realizados en $2010(\mathrm{n}=38)$ fue detectada la presencia de $P$. collybita y $P$. ibericus. Con estos datos hemos de afirmar que nuestros resultados únicamente se corresponden con aquellos publicados por BALMORI et $A L$. (2002), ya que si ellos sólo detectaron la presencia de $P$. collybita/ibericus en el 4,8\% de los recorridos que realizaron, en nuestro caso la cifra también es muy parecida $(6,4 \%)$. 


\section{CONSIDERACIONES FINALES}

La conclusión general de nuestro trabajo preliminar es que tanto $P$. ibericus como $P$. collybita pueden considerarse especies reproductoras muy escasas en la provincia de Salamanca, con una distribución muy irregular y dispersa, ya que en principio sólo concurren en áreas con ombroclima húmedo y en bosques de ribera con estructura compleja, con presencia variable según años y cambiante según zonas.

A pesar de que varios estudios previos comentados en el texto acentúen las exigencias ambientales de ambas especies (ambientes montanos, forestales, con temperaturas suaves y precipitaciones abundantes, o bien bosques de ribera), el bajo número de contactos obtenidos, así como los resultados publicados por otros autores, que informan acerca de una distribución más amplia de ambas especies en la provincia de Salamanca, aconsejan la realización de futuras prospecciones y la revisión de los datos que se vayan incorporando a la base de datos SACRE. En este sentido deberíamos remarcar que si consideramos los umbrales bioclimáticos utilizados por HUNTLEY et AL. (2007) para definir las zonas potenciales de $P$. ibericus/collybita en el continente europeo (que el ratio entre evapotranspiración real y evapotranspiración potencial sea igual o mayor que 0,6 y que los días del año con temperaturas superiores a $5^{\circ} \mathrm{C}$ sumen más de 200 ), la mayor parte del territorio de la provincia de Salamanca potencialmente podría acoger ambas especies.

De acuerdo con trabajos como el de STERLING (1996), que explican la capacidad de los bosques de ribera para crear unas condiciones microclimáticas específicas que los habilitan para actuar como refugio de especies de distribución eurosiberiana, nuestra hipótesis de trabajo es que el bioclima determina la ocurrencia espacial de hábitats para ambas especies de mosquiteros y que los bosques de ribera complejos, con su característica componente azonal, pueden albergar ejemplares en escaso número en zonas de bioclima desfavorable. No obstante planteamos nuevas campañas de campo con el objetivo de:

1) seguir prospectando bosques riparios de estructura compleja en zonas con bioclima seco y subhúmedo.

2) seguir prospectando las zonas forestales con bioclima húmedo en el sur de la provincia.

3) seguir prospectando de forma selectiva algunos hábitats forestales que no cumplen las características anteriores (por ejemplo, melojares y quejigares subhúmedos, dehesas de encinar castellano o bosques isla en las "estepas" cerealistas) para contrastar los resultados de algunos atlas y estudios de tendencia poblacional (PERIS y CARNERO, 1988; 
Cuesta et AL., 2003; CARRASCAL y PALOMINO, 2008) que amplían el rango de hábitats de $P$. ibericus y $P$. collybita.

\section{Agradecimientos:}

Sin la colaboración de una serie de personas e instituciones no hubiera sido posible la realización del presente trabajo, debido a lo cual queremos manifestar nuestro más sincero agradecimiento a Eloisa Matéu (Alosa Sonidos de la Naturaleza) por la cesión gratuita de numerosas pistas con cantos de Phylloscopus ibericus y Phylloscopus collybita, a SEO/BirdLife por facilitarnos el acceso a los datos sobre ambas especies en la provincia de Salamanca, incluidos en la base de datos del programa SACRE, y a Alfonso Balmori por su valiosa revisión crítica del documento original.

\section{REFERENCIAS BIBLIOGRÁFICAS}

BAKER, K. (1997): Warblers of Europe, Asia and North Africa. Christopher Helm, A \& C Black, London. 400 pp.

Balmori, A.; Cuesta, M. A.; CABAllero, J. M. (2002): «Distribución de los mosquiteros ibérico (Philloscopus brehmii) y europeo (Phylloscopus collybita) en los bosques de ribera de Castilla y León (España)». Ardeola, 49 (1): 19-27.

BAlmori, A. y Cuesta, M. A. (2003): «Distribución del Mosquitero Ibérico (Phylloscopus ibericus) y el Mosquitero Común (Phylloscopus collybita) en la Península Ibérica». El Escribano Digital, 37: 6.

Blanco, M. (Coord.) (2004): Anuario Ornitológico de la provincia de Salamanca, 1924-2003. SEO-Salamanca. 139 pp.

Blanco, M. (Coord) (2007): Anuario Ornitológico de la provincia de Salamanca, 2004-2006. SEO-Salamanca. 178 pp.

BuENo HeRnÁnDEZ, R. (2000): Las aves de la sierra de Béjar. Edición a cargo del autor. Madrid. 222 pp.

CARRASCAL L. M. y PALOMINO, D. (2008): Las aves comunes reproductoras en España. Población en 2004-2006. SEO/BirdLife, Madrid. 202 pp.

Catry, P.; Lecoq, M. Araújo, A. Conway, G. Felgueiras, M. J.; King, M. B.; Rumsey, S. SAlima, H. y TenREIRO, P. (2005): «Differential migration of chiffchaffs Phylloscopus collybita and P. ibericus in Europe and Africa». Journal of Avian Biology, 36: 184-190.

CRAMP, S. (1992): Handbook of the birds of Europe, the Middle East and North Africa. Vol. 6. Oxford University Press. Oxford.

Cuesta, M. A. y BAlmori, A. (2003a): «Mosquitero Común y Mosquitero Ibérico. Phylloscopus collybita y P. ibericus». En R. MARTí y J. C. DEL MORAL (Eds): Atlas de las Aves Reproductoras de España, pp. 488-491. Dirección General de Conservación de la Naturaleza-Sociedad Española de Ornitología. Madrid.

De JuAnA, E. y VARela, J. (2000): Guía de las aves de España. Lynx Edicions, Barcelona. 223 pp. 
Del Hoyo, J. ElliotT, A. y Christie, D.A. eds. (2006): Handbook of the birds of the world. Vol. 11. Old world flycatchers to old world warblers. Lynx Edicions, Barcelona. $798 \mathrm{pp}$.

EliAS, G. (2004): «Aspects of Iberian Chiffchaff Phylloscopus ibericus distribution in Spain and Portugal». Ibis, 146: 685-686.

Helbig, A.J.; Martens, J.; Seibold, I.; Henning, F.; Schotler, B. y Wink, M. (1996): «Phylogeny and species limits in the Paleartic chiffchaff Phylloscopus collybita complex: mitochondrial differentiation and bioacustic evidence». Ibis, 138: 650-666.

Huntley, B.; Green, R. E.; Collingham, Y. C. y Willis, S. G. (2007): A Climatic Atlas of European Breeding Birds. Durham University; The RSPB and Lynx Edicions. Barcelona. 521 pp.

MCELwEE, A. (2010): «Identification of an Iberian Chiffchaff in Norfolk». Birding World, 23 (4): 150-153.

OnRUBia, A.; Arroyo, J.L.; ANDRÉS, T.; GómeZ, J.; UnAmunO, J.M. y ZuFIAUR, F. (2003): «El mosquitero ibérico (Phylloscopus ibericus): identificación, biometría y apuntes sobre su migratología». Revista de Anillamiento, 12: 18-29.

PERIS, S.J. y CARNERO, J. (1988): Atlas ornitológico de la provincial de Salamanca. Ediciones de la Diputación de Salamanca, Salamanca. 244 pp.

Rivas-MARTíneZ, S. (1987): Memoria del mapa de las series de vegetación de España. Ed. ICONA, Madrid.

SAlOMON, M.; VoISIN, J-F. y BRIED, J. (2003): «On the taxonomic status and denomination of the Iberian Chiffchaffs». Ibis, 145: 87-97.

SAnZ-ZuASTI, J. y Velasco, T. Guía de las aves de Castilla y León. Nueva edición revisada y ampliada. Náyade Editorial, Medina del Campo (VA). 407 pp.

STERLING, A. (1996): Los sotos, refugio de vida silvestre. Ministerio de Agricultura, Pesca y Alimentación. Secretaría General Técnica, Madrid. 266 pp.

Svensson, L.; Mullarney, K. y ZetTerström, D. (2010): Collins Bird Guide 2nd Edition. Harper Collins, London.

Tellería, J.L.; Asensio, B. y DíAZ, M. (1999): Aves ibéricas II. Paseriformes. J.M. Reyero Ed. Madrid.

\section{Páginas WEB:}

Programa SACRE (Seguimiento de las Aves Comunes Reproductoras en España), dirigido por SEO/BirdLife: http://www.seo.org/programa_seccion_ficha.cfm?Id Programa $=3 \&$ idArticulo $=223$ 